\title{
Defective responsiveness to ascorbic
of neutrophil random and chemotactic migration in Felty's syndrome and systemic lupus erythematosus
}

\author{
EDWARD J. GOETZL
}

From the Departments of Medicine, Harvard Medical School and Robert B. Brigham Hospital, Boston, Massachusetts, U.S.A.

\begin{abstract}
Goetzl, E. J. (1976). Annals of the Rheumatic Diseases, 35, 510-515. Defective responsiveness to ascorbic acid of neutrophil random and chemotactic migration in Felty's syndrome and systemic lupus erythematosus. Polymorphonuclear (PMN) leucocytes from 4 patients with untreated systemic lupus erythematosus (SLE) showed defective random migration $(P<0.05)$ and depressed chemotactic responses to $\mathrm{C5a}$ and kallikrein $(\mathbf{P}<0.01)$ compared to $P M N$ leucocytes from normal subjects, or patients with rheumatoid arthritis (4) or Felty's syndrome (4) when examined at a standardized cell concentration with a micropore filter radioassay but not with a conventional Boyden technique. Normal in vitro enhancement of PMN leucocyte random and chemotactic migration by sodium ascorbate was absent in SLE and Felty's syndrome, but sodium ascorbate gave normal stimulation of hexose monophosphate shunt activity in the PMN leucocytes precluding a defect in ascorbate transport.
\end{abstract}

The high incidence of focal and systemic bacterial and other infections in patients with systemic lupus erythematosus (SLE) and Felty's syndrome has been attributed in part to a deficiency in the polymorphonuclear (PMN) leucocyte component of host defence (Shearn and Pirofsky, 1952; Ruderman, Miller, and Pinals, 1968; Estes and Christian, 1971; Dubois, 1974; Staples and others, 1974). A poor correlation between the risk of infection and the extent of peripheral blood leucopenia in such patients has led to the postulation of a qualitative defect in the PMN leucocytes (Louise and Pearson, 1971; Mowat, 1972; Staples and others, 1974).

Several studies have documented distinct abnormalities of phagocytosis and nitroblue tetrazolium reduction by PMN leucocytes in SLE (Brandt and Hedberg, 1969; Orozco, Jasin, and Ziff, 1970; Wenger and Bole, 1973) and low in vitro PMN leucotactic responsiveness to a chemotactic factor in rheumatoid arthritis, Felty's syndrome, and in some patients with SLE (Mowat and Baum, 1971; Zivkovic and Baum, 1972; Clark, Kimball, and Decker, 1974). In addition to analysing the erythrophagocytic capacity and random and $\overrightarrow{\vec{\rho}}$ chemotactic migration of PMN leucocytes in 3 rheumatic states, this study correlates the results from two distinct in vitro assays of migration and employs ascorbate stimulation of PMN leucocyte migration as a probe for intrinsic cell defects.

\section{Materials and methods}

Thirteen adult patients were evaluated, of whom 4 have seropositive rheumatoid arthritis (RA), 1 has vasculitis, 0 4 have Felty's syndrome, and 4 have SLE. An age- and sex-matched normal subject was studied concurrently with each patient to provide 13 control analyses of leucocyte migration. All patients were inpatients or out- $N$ patients at the Peter B. Brigham Hospital or Robert B. Brigham Hospital and received a comprehensive general 0 medical evaluation at the time of the leucocyte studies. $\underset{W}{ }$ Diagnoses were based on standard criteria (Ruderman 0 and others, 1968; Louise and Pearson, 1971; Trimble and others, 1974), and all patients with RA, SLE, and Felty's syndrome had active disease. The patient with vasculitis $\mathscr{D}$ had a necrotizing cutaneous angiitis documented by a ? characteristic skin biopsy and was free of joint or other organ system involvement. All of the patients with $\underset{\mathbb{\mathrm { D }}}{\mathrm{O}}$ 
Felty's syndrome and 2 of the 4 with SLE exhibited peripheral blood leucopenia at the time of the study. None of the patients had azotaemia, liver disease, or diabetes mellitus. Each patient was receiving aspirin in doses ranging from $0.6-1.8 \mathrm{~g} /$ day but had not taken any other anti-inflammatory medication or any drug known to influence PMN leucocyte function in the 3month period preceding evaluation.

Polystyrene disposable chemotactic chambers (Adaps, Dedham, Mass.) and acrylic radiochemotactic chambers (Neuroprobe, Bethesda, Md.) were assembled with $3 \mu \mathrm{m}$ and $8 \mu \mathrm{m}$ pore size micropore filters (Millipore, Bedford, Mass.) as previously described (Goetzl and Austen, 1972a, b). Hanks's solution and medium 199 with or without ascorbic acid (Microbiological Associates, Bethesda, Md.), ovalbumin five times recrystallized (Miles-Seravac, Kankakee, Ill.) dextran, Sephadex and Ficoll (Pharmacia Laboratories, Piscataway, N.J.), sodium diatrizoate (Hypaque, Winthrop Laboratories, New York, N.Y.), two times recrystallized trypsin and soybean trypsin inhibitor (Worthington Biochemical, Freehold, N.J.), sodium ${ }^{51} \mathrm{Cr}$-chromate, $1{ }^{14} \mathrm{C}$-glucose and $6-{ }^{14} \mathrm{C}$-glucose (Amersham-Searle, Arlington Heights, Ill.), sodium lauryl sulphate and ascorbic acid (Fisher Scientific, Medford, Mass.), and plastic 35- $\times 10-\mathrm{mm}$ Petri dishes (Falcon Plastics, Oxnard, Calif.) were obtained. Gamma radiation from ${ }^{51} \mathrm{Cr}$-containing micropore filters was measured with a dual channel gamma well counter, and $\beta$-radiation from ${ }^{14} \mathrm{C}$-glucose solutions was quantitated with Bray's fluid in a liquid scintillation counter (NuclearChicago, Des Plaines, Ill.).

ASSESSMENT OF CHEMOTAXIS, RANDOM MIGRATION, AND PHAGOCYTOSIS

Chemotaxis of human leucocytes was assayed by a modification (Goetzl and Austen, 1972a) of the Boyden micropore filter technique (Boyden, 1962) and by a radiochemotactic method using modified chambers that hold a layer of two closely apposed micropore filters between ${ }^{51} \mathrm{Cr}$-labelled leucocytes and the chemotactic stimulus (Goetzl and Austen, 1972b). Human peripheral leucocytes from normal subjects and patients were collected and separated from erythrocytes by dextran sedimentation (Goetzl and Austen, 1972a), and the neutrophil PMN leucocyte fraction was purified by centrifugation of the mixed leucocytes on Ficoll-Hypaque cushions (Böyum, 1968).

Neutrophils were washed twice and resuspended in ascorbate-free medium 199 with $0.4 \mathrm{~g}$ ovalbumin $/ 100 \mathrm{ml}$ (pH 7.4) for single micropore filter chambers of the modified Boyden technique or medium 199 without added protein for ${ }^{51} \mathrm{Cr}$ labelling (Goetzel and Austen, 1972a, b). The ${ }^{51} \mathrm{Cr}$-labelled neutrophils were washed twice and resuspended in ascorbate-free medium 199ovalbumin. The neutrophil suspensions were adjusted to a standard range of concentrations of $2 \cdot 2 \pm 0.4 \times 10^{8} / \mathrm{ml}$ in the modified Boyden method with a $3 \mu \mathrm{m}$ pore filter for the chamber and $5.0 \pm 0.5 \times 10^{6} / 0.5 \mathrm{ml}$ containing 50 000-70 000 counts per 4 minutes (CP $4 \mathrm{~min}$ ) for the radioassay with an $8 \mu \mathrm{m}$ pore filter over a $3 \mu \mathrm{m}$ pore filter for the chamber.

The chemotactic factors were $\mathrm{C} 5 \mathrm{a}$ generated by tryptic digestion of highly purified human C5 (Ward and Newman, 1969) and human kallikrein produced from purified pre- kallikrein by the action of Hageman factor fragments (Kaplan, Kay, and Austen, 1972). Random migration in the absence of a chemotactic factor was assessed with the modified Boyden method by increasing the incubation interval from the $2 \frac{1}{2}$-hour chemotactic period to $3 \frac{1}{2}$ hours with the radioassay by increasing the interval from the 4-hour chemotactic period to 5 hours at $37^{\circ} \mathrm{C}$.

The neutrophil response in the modified Boyden assay was quantitated by counting the cells in fixed and stained filters at $80 \mu \mathrm{m}$ from their source at the top of the filter and was expressed as leucocytes per high power field (hpf) with a correction for background migration in the chemotactic assay. The neutrophil response in the radioassay was calculated as CP $\mathbf{4} \mathrm{min}$ in the lower filter per $50000 \mathrm{CP} 4 \mathrm{~min}$ in the inital neutrophil suspension with a correction for background migration in the chemotactic assay. The enhancement of random or chemotactic migration of neutrophils preincubated for 10 minutes and assayed with added sodium ascorbate was expressed as a per cent of unstimulated migration (Goetzl and others, 1974). Statistical analyses used a standard two-sample $t$ test.

Phagocytosis by human neutrophils of antibodysensitized erythrocytes coated with $\mathrm{C} 3 \mathrm{~b}$ as a result of interaction with the first four components of complement was determined as described (Gigli and Nelson, 1968). Human neutrophils at a concentration of $3-4 \times 10^{8} / \mathrm{ml}$ were incubated with $2 \times 10^{7}$ target cells for 20 minutes at $37^{\circ} \mathrm{C}$, and the per cent phagocytosis in duplicate tubes was calculated by subtracting the absorbancy units at $414 \mathrm{~nm}$ (AU 414) of $0.84 \% \mathrm{NH}_{4} \mathrm{Cl}$ lysates of the uningested erythrocytes in phagocytosis mixtures from the AU 414 of a lysate of total initial erythrocytes and dividing this difference by the latter AU 414 (Gigli and Nelson, 1968).

MEASUREMENT OF HEXOSE MONOPHOSPHATE SHUNT (HMPS) ACTIVITY

The HMPS activity of layers of neutrophils adherent to plastic Petri dishes was measured by the rate of generation of ${ }^{14} \mathrm{CO}_{2}$ from $1{ }^{14} \mathrm{C}$-glucose as previously described (Goetzl and Austen, 1974). 3-4 × $10^{6}$ neutrophils adhered to each Petri dish, and the counts per minute (cpm) of ${ }^{14} \mathrm{CO}_{2}$ generated over 80 minutes at $37^{\circ} \mathrm{C}$ was standardized by dividing by the $\mathrm{AU}$ at $280 \mathrm{~nm}$ of a sodium lauryl sulphate solution of the adherent cells. HMPS activity was expressed as cpm/0.2 AU 280.

\section{Results}

The assessment of in vitro neutrophil random migration with the radioassay showed significantly depressed values relative to those of normal neutrophils only for the cells of patients with SLE $(P<$ $0.05)$, which was not appreciated $(P=0.05)$ in the modified Boyden assay (Fig. 1). The radioassay was far more sensitive than the modified Boyden assay in unveiling a significant in vitro defect in chemotactic responsiveness of neutrophils from SLE patients to either C5a $(P<0.01)$ (Fig. 2) or kallikrein. SLE patients with leucopenia had neutrophil migration values comparable to those for the SLE patients with normal leucocyte levels. Neutrophils from the 


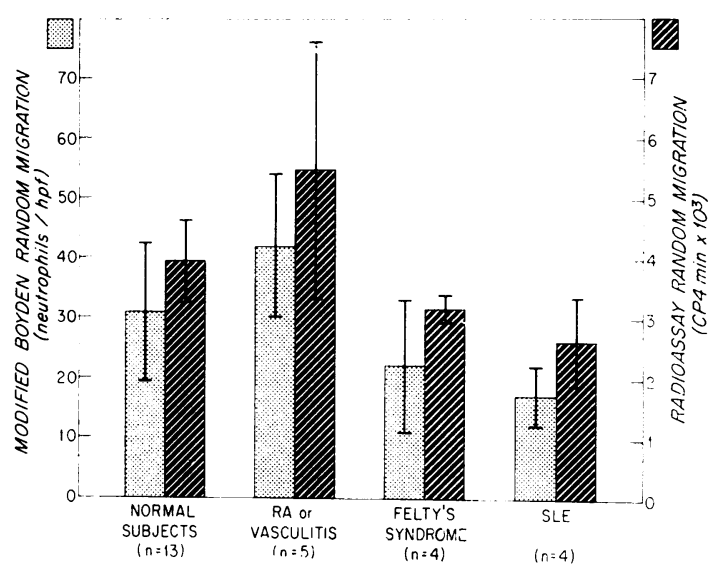

FIG. 1 Neutrophil random migration. The bars show mean $\pm 1 S D$ for each group. CP 4-min values for the radioassay are expressed per $50000 \mathrm{CP} 4 \mathrm{~min}$ in the initial ${ }^{51} \mathrm{Cr}$-labelled neutrophil suspension. Random migration was significantly depressed in the radioassay for SLE patients only $(P<0.05)$

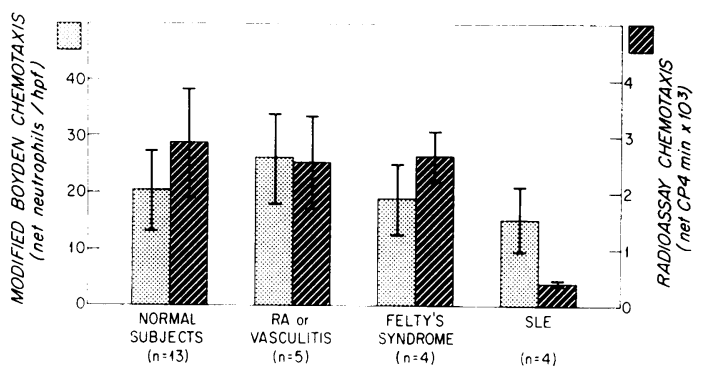

FIG. 2 Neutrophil chemotaxis. The bars show mean \pm $1 S D$ for each group. The standardized C5a stimulus was generated by tryptic digestion of $2.5 \mu \mathrm{g}$ of purified human C5 per chamber. Chemotaxis was significantly depressed in the radioassay for $S L E$ patients only $(P<0.01)$

patients with Felty's syndrome, rheumatoid arthritis (RA), or vasculitis exhibited random migration and chemotactic responsiveness to $\mathrm{C} 5 \mathrm{a}$ and kallikrein that were indistinguishable from normal levels in both assays.

The ability of sodium ascorbate to stimulate random and chemotactic migration of human leucocytes by an action on the cells was used to confirm the migration defect in SLE. Ascorbate at a concentration of $2.5 \times 10^{-3} \mathrm{~mol} / 1$ failed in either assay to enhance the random migration (Fig. 3) or chemotactic responsiveness to C5a (Fig. 4) or to kallikrein of neutrophils from patients with SLE or Felty's syndrome. Ascorbate enhancement of random and chemotactic migration of neutrophils from normal subjects or patients with RA or vasculitis in an identical protocol averaged $55-95 \%$ of baseline migration.

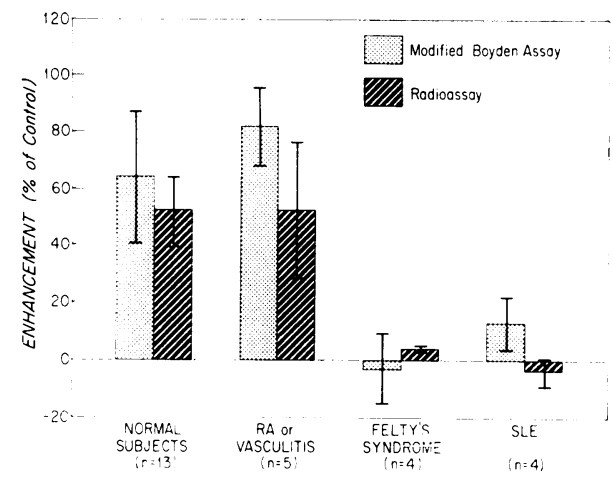

FIG. 3 Ascorbate enhancement of neutrophil random migration. The bars show mean $\pm 1 S D$ for each group. The ascorbate dose of $2.5 \times 10^{-3} \mathrm{~mol} / \mathrm{l}$ was used with a 10-min preincubation interval

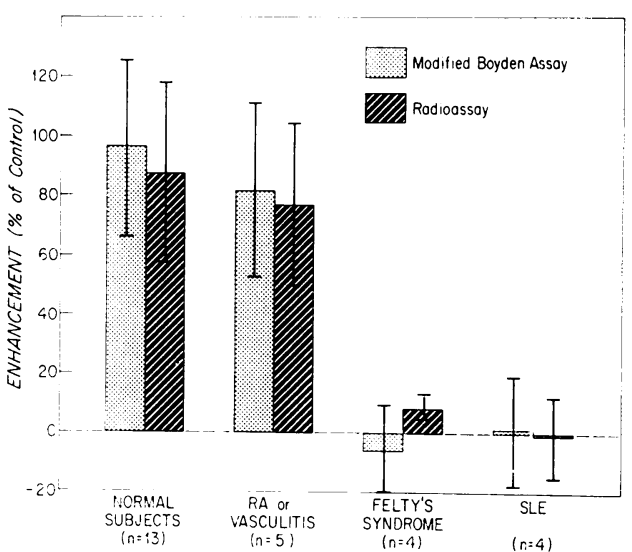

FIG. 4 Ascorbate enhancement of neutrophil chemotaxis. The bars show the mean $\pm 1 S D$ for each group. The ascorbate dose of $2.5 \times 10^{-3} \mathrm{~mol} / \mathrm{l}$ was used with a 10-min preincubation interval

Since the ascorbate enhancement of migration requires a concomitant stimulation of leucocyte HMPS activity (Goetzl and others, 1974b), the influence of increasing concentrations of ascorbate on both functions was examined in parallel studies (Fig. 5). A dose-related stimulation of random migration of neutrophils from two normal subjects was observed in modified Boyden chambers. Random migration of neutrophils from 2 patients with Felty's syndrome was first enhanced detectably at an ascorbate concentration of $5 \times 10^{-3} \mathrm{~mol} / \mathrm{l}$ and required an 8-fold higher ascorbate level for a rise to the study enhancement range (Figs. 3, 5). In contrast, ascorbate produced a similar doseresponse stimulation of neutrophil HMPS activity for both normal controls and patients with Felty's syndrome (Fig. 5). Studies of neutrophil phagocytosis of antibody-sensitized C3b-coated erythrocytes 


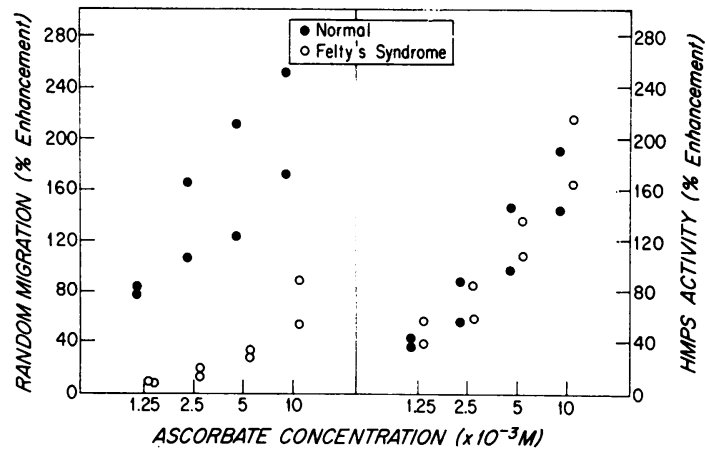

FIG. 5 Dose-response of ascorbate enhancement of neutrophil random migration and hexose monophosphate shunt (HMPS) activity. The preincubation interval for ascorbate was 5 min. Baseline HMPS activity was 631 and $479 \mathrm{cpm}$ per $0.2 \mathrm{AU} U_{280}$ for the 2 normal subjects and 532 and $327 \mathrm{cpm}$ per $0.2 A U_{280}$ for the 2 patients with Felty's syndrome. Unstimulated random migration in the modified Boyden assay was 24 and 28 neutrophils/hpf for normal subjects, and 17 and 23 neutrophils/hpf for patients with Felty's syndrome

showed no significant differences between control subjects who had a range of $68 \cdot 3-84 \cdot 1 \%$ phagocytosis, patients with RA or vasculitis, with a range of $71.9-87.5 \%$ phagocytosis, and patients with Felty's syndrome or SLE, who had a range of $64 \cdot 7-82 \cdot 6 \%$ phagocytosis.

\section{Discussion}

Neutrophil PMN leucocytes from patients with SLE exhibited a significant defect in random migration and chemotaxis in response to the purified stimuli, C5a and kallikrein, as assessed in a micropore filter radioassay (Figs. 1, 2). In contrast, the radioassay of random migration and chemotaxis of neutrophils from patients with Felty's syndrome, RA, or vasculitis gave levels indistinguishable from those of neutrophils from normal subjects. Pretreatment with sodium ascorbate of neutrophils from patients with SLE or Felty's syndrome failed to stimulate their random migration or chemotactic responsiveness, whereas there was a $55-95 \%$ enhancement for neutrophils of control subjects and patients with RA or vasculitis (Figs. 3, 4). Thus, neutrophils of patients with RA or vasculitis were normal with respect to their capacity for phagocytosis of opsonized erythrocytes, random and chemotactic migration, and ascorbate enhancement of migration. Neutrophils of patients with SLE showed impaired baseline random and chemotactic migration and depressed responsiveness of migration to ascorbate, but normal phagocytosis. Patients with Felty's syndrome were an intermediate group since both baseline migration and phagocytic capacity of neutrophils were normal, but ascorbate failed to enhance random or chemotactic migration.

The finding of normal neutrophil phagocytosis in RA agrees with previous studies (Brandt and Hedberg, 1969). A defect in phagocytosis by neutrophils from patients with SLE (Brandt and Hedberg, 1969; Orozco and others, 1970) has been attributed to a serum deficiency which can be reversed with normal serum (Orozco and others, 1970). The current erythrophagocytosis assay, which yielded normal values for neutrophils in SLE, uses previously opsonized C3b-coated erythrocytes and thus would not detect defects other than those inherent to the neutrophils. Abnormally low chemotactic indices have been noted with modified Boyden assays for patients with RA and Felty's syndrome, while most patients with SLE had a normal chemotactic index (Mowat and Baum, 1971; Zivkovic and Baum, 1972; Clark and others, 1974). The modified Boyden assay did not show a significant defect in neutrophil migration in any of the patient groups (Figs. 1, 2). The radioassay, which imposes a double micropore filter barrier, clearly unveiled the intrinsic leucotactic abnormality in SLE. Further differences in both methodology and patient populations exist between this study and previous investigations. The current assays used in vitro standardization of the concentration of neutrophil suspensions in contrast to the mathematical approach used to correct for differences in initial cell counts in some studies (Zivkovic and Baum, 1972). Finally, the majority of previously reported SLE patients were receiving moderate to high dose corticosteroid therapy at the time of migration studies (Zivkovic and Baum, 1972; Clark and others, 1974), which can be associated with improvement of the in vitro chemotactic index in SLE and other diseases (Mowat and Baum, 1971).

The strikingly decreased responsiveness to ascorbate of neutrophil migration in Felty's syndrome and SLE was dose-related with moderate enhancement of migration at high concentrations of ascorbate (Fig. 5). The reduced responsiveness of neutrophil migration to ascorbate with full stimulation by ascorbate of HMPS activity in dose-response studies precludes a defect in ascorbate intracellular transport or metabolic utilization (Fig. 5). Stimulation of HMPS activity without enhancement of neutrophil migration has previously been observed with methylene blue (Goetzl and others, 1974b). HMPS stimulation by a chemotactic factor or ascorbate may be associated with inhibition of the neutrophil migration response to the same stimuli when the test cells have been deactivated by prior exposure to a chemotactic principle or previously treated with the neutrophil immobilizing factor (NIF) or the 
basic tetrapeptide, Thr-Lys-Pro-Arg (Goetzl and others, 1973; Goetzl and Austen, 1974; Goetzl, 1975).

Deactivation of leucocytes by prior exposure to a chemotactic factor exhibits a dose-related leucocyte specificity that parallels the specificity of chemotactic stimulation by each principle (Goetzl, Wasserman, and Austen, 1974a; Goetzl, Schreiber, and Austen, 1976) and is dependent upon the stimulation and subsequent decay of a leucocyte esterase (Ward and Becker, 1968). NIF, a non-chemotactic 4000-5000 molecular weight basic peptide derived from leucocyte granules, inhibits the migration of polymorphonuclear leucocytes with no effect on phagocytosis or mononuclear leucocyte migration (Goetzl and Austen, 1972a; Goetzl and others, 1973). The leucocyte phagocytosis-stimulating basic tetrapeptide, Thr-Lys-Pro-Arg, is liberated by enzymatic digestion of a fraction of human $\gamma$-globulin and, like NIF, inhibits migration of PMN leucocytes without influencing mononuclear leucocyte migration (Nishioka and others, 1972; Goetzl, 1975).

Prior exposure of neutrophils to a chemotactic factor so as to induce deactivation, or to NIF or Thr-Lys-Pro-Arg tetrapeptide, results in inhibition of both random and chemotactic migration and blunts enhancement of migration by ascorbate without blocking the stimulation of HMPS activity. Thus, the isolated hyporesponsiveness to ascorbate of neutrophils in Felty's syndrome and the more extensive defect in both baseline and ascorbatestimulated migration in SLE may result from exposure of the cells to one or more humoral inhibitors elaborated in an area of inflammatory reaction (Goetzl, 1975). The defect in neutrophils in SLE could be caused by either chemotactic factor deactivation or by an encounter with NIF or the ThrLys-Pro-Arg tetrapeptide. The uniquely isolated \& lack of ascorbate responsiveness of neutrophil $\vec{\circ}$ migration in Felty's syndrome is not reminiscent of the effect of any one humoral inhibitor but may result from low levels of several inhibitors acting in concert.

Analysis of the precise relationship of the neutrophil migration defects in rheumatic diseases to individual inhibitors must await their further purification, and the development of techniques to measure their concentrations in serum and synovial fluid. Nonetheless, the presence of qualitative defects in neutrophil function, in addition to quantitative deficiencies, may contribute in part to impaired host resistance to infection.

Supported by grants AI-07722 and AI-10356 from the National Institutes of Health. E. J. G. is Director of th8 Laboratories for the Study of Immunological Diseases of the Howard Hughes Medical Institute.

\section{References}

BOYDEN, S. (1962) J. exp. Med., 115, 453 (The chemotactic effect of mixtures of antibody and antigen on polymorphonuclear leukocytes)

BöyuM, A. (1968) Scand. J. clin. Lab. Invest., 21, Suppl. 97, 31 (Isolation of leukocytes from human blood; further observations)

BRANDT, L., AND HedBeRG, H. (1969) Scand. J. Haemat., 6, 348 (Impaired phagocytosis by peripheral blood granulocytes in systemic lupus erythematosus)

Clark, R. A., Kimball, H. R., AND Decker, J. L. (1974) Ann. rheum. Dis., 33, 167 (Neutrophil chemotaxis in systemic lupus erythematosus)

Duвors, E. L. (1974) 'The clinical picture of systemic lupus erythematosus' in 'Lupus Erythematosus', 2nd ed., p 435. University of Southern California Press, Los Angeles

Estes, D., AND Christian, C. L. (1971) Medicine, 50, 85 (The natural history of systemic lupus erythematosus by prospective analysis)

Gigli, l., AND Nelson, R. A., JR. (1968) Exp. cell Res., 51, 45 (Complement dependent immune phagocytosis. I. Requirements for $\mathrm{C} 1, \mathrm{C} 4, \mathrm{C} 2, \mathrm{C} 3)$

GoetZL, E. J. (1975) Ann. N.Y. Acad. Sci., 256, 210 (Plasma and cell-derived inhibitors of human neutrophil chemotaxis)

—, AND AUSTEN, K. F. (1972a) J. exp. Med., 136, 1564 (A neutrophil immobilizing factor derived from human leukocytes. I. Generation and partial characterization)

- - - - (1972b) Immunol. Commun., 1, 421 (A method for assessing the in vitro chemotactic response of neutrophils utilizing ${ }^{51} \mathrm{Cr}$-labeled human leukocytes)

- - _ - (1974) J. clin. Invest., 53, 591 (Stimulation of human neutrophil leukocyte aerobic glucose metabolism by purified chemotactic factors)

-, WASSERMAN, S. I., AND AUSTEN, K. F. (1974a) 'Modulation of the eosinophil chemotactic response in immediate hypersensitivity' in 'Progress in Immunology II', eds. L. Brent and J. Holborow, Vol. 4, p. 41. NorthHolland, Amsterdam

- SChreiber, A. D. AND Austen, K. F. (1976) In 'Chemotactic Activity of Components of the Kallikreinkinin System in Health and Disease', ed. J. Pisano. National Institutes of Health, Bethesda, Maryland (in press) 
—, Gigli, I., Wasserman, S. I., AND Austen, K. F. (1973) J. Immunol, 111, 938 (A neutrophil immobilizing factor derived from human leukocytes. II. Specificity of action on polymorphonuclear leukocyte mobility)

-, Wasserman, S. I., Gigli, I., AND Austen, K. F. (1974b) J. clin. Invest., 53, 813 (Enhancement of random migration and chemotactic response of human leukocytes by ascorbic acid)

KaPlan, A. P., KAY, A. B., AND Austen, K. F. (1972) J. exp. Med., 135, 81 (A prealbumin activator of prekallikrein. III. Appearance of chemotactic activity for human neutrophils by the conversion of human prekallikrein to kallikrein)

Louise, J. S., AND Pearson, C. M. (1971) Semin. Hematol., 8, 216 (Felty's syndrome)

Mowat, A. G. (1972) Semin. Arthr. Rheum., 3, 195 (Hematologic abnormalities in rheumatoid arthritis)

-, AND BAUM, J. (1971) J. clin. Invest., 50, 2541 (Chemotaxis of polymorphonuclear leukocytes from patients with rheumatoid arthritis)

Nishoka, K., Constantopoulos, A., Satoh, P. S., and Najjar, V. A. (1972) Biochem. biophys Res. Commun., 47, 172 (The characteristics, isolation and synthesis of the phagocytosis stimulating peptide Tuftsin)

Orozco, J. H., JASIN, H. E., AND ZIFF, M. (1970) Arthr. and Rheum., 13, 342 (Defective phagocytosis in patients with systemic lupus erythematosus)

Ruderman, M., Miller, L. M., And Pinals, R. S. (1968) Ibid., 11, 377 (Clinical and serologic observations on 27 patients with Felty's syndrome)

Shearn, M. A., AND PIROFSKy, B. (1952) Arch. intern. Med., 90, 790 (Disseminated lupus erythematosus: analysis of 34 cases)

Staples, P. J., Gerding, D. N., DeCKer, J. L., AND GoRdon, R. S., JR. (1974) Arthr. and Rheum., 17, 1 (Incidence of infection in systemic lupus erythematosus)

Trimble, R. B., Townes, A. S., Robinson, H., Kaplan, S. B., Chandler, R. W., Hanissian, A. S., and Masi, A. T. (1974) Ibid., 17, 184 (Preliminary criteria for the classification of SLE: evaluation in early diagnosed SLE and rheumatoid arthritis)

WARD, P. A., AND BECKER, E. L. (1968) J. exp. Med., 127, 693 (The deactivation of rabbit neutrophils by chemotactic factor and the nature of the activatable esterase)

-. AND NEWMAN, L. J. (1969) J. Immunol., 102, 93 (A neutrophil chemotactic factor from human C5)

WeNGeR, M. E., AND Bole, G. G. (1973) J. Lab. clin. Med., 82, 513 (Nitroblue tetrazolium (NBT) dye reduction by peripheral leukocytes from patients with rheumatoid arthritis and systemic lupus erythematosus measured by a histochemical and spectrophotometric method)

Zivkovic, M., AND BAUM, J. (1972) Immunol. Commun., 1, 39 (Chemotaxis of polymorphonuclear leukocytes from patients with systemic lupus erythematosus and Felty's syndrome) 